\title{
Left ventricular reshaping: Effects on the pressure-volume relationship
}

\author{
Abul Kashem, MD, PhD \\ Sarmina Hassan, MD, $\mathrm{PhD}^{\mathrm{a}}$ \\ Deborah L. Crabbe, $\mathrm{MD}^{\mathrm{a}}$ \\ David B. Melvin, MD ${ }^{\mathrm{b}}$ \\ William P. Santamore, $\mathrm{PhD}^{\mathrm{a}}$
}

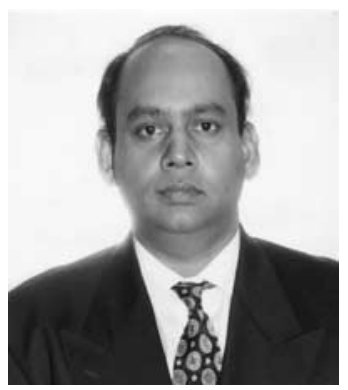

Dr Kashem

\footnotetext{
From Cardiovascular Research, Temple University, Philadelphia, $\mathrm{Pa},{ }^{\mathrm{a}}$ and the Department of Surgery, University of Cincinnati, Cincinnati, Ohio. ${ }^{\mathrm{b}}$

Supported by a grant from CardioClasp, Inc.

David B. Melvin, MD, is the inventor of CardioClasp device and owns indirect equity in CardioClasp, Inc, Cincinnati, Ohio; William P. Santamore, $\mathrm{PhD}$, is the Consultant for the CardioClasp, Inc; and Abul Kashem, $\mathrm{MD}, \mathrm{PhD}$, is the institutional principal investigator for the CardioClasp device.

Read at the Eighty-second Annual Meeting of The American Association of Thoracic Surgery, Washington, DC, May 5-8, 2002.

Received for publication June 4, 2002; revisions requested July 22, 2002; revisions received July 31, 2002; accepted for publication Aug 2, 2002.

Address for reprints: Abul Kashem, MD, $\mathrm{PhD}$, Temple University School of Medicine, Medical Research Building, Room 800A, 3420 N. Broad St, Philadelphia, PA 19140 (E-mail: mkashem@temple.edu).

J Thorac Cardiovasc Surg 2003;125:391-9

Copyright (C) 2003 by The American Association for Thoracic Surgery

$0022-5223 / 2003 \$ 30.00+0$

doi: $10.1067 / \mathrm{mtc} .2003 .4$
}

Objective: We tested whether the CardioClasp device (CardioClasp, Inc, Cincinnati, Ohio), a non-blood contact device, would improve left ventricular contractility by acutely reshaping the left ventricle and reducing left ventricular wall stress.

Methods: In dogs $(n=6) 4$ weeks of ventricular pacing $(210-240 \mathrm{ppm})$ induced severe heart failure. Left ventricular function was evaluated before and after placement of the CardioClasp device, which uses 2 indenting bars to reshape the left ventricle. Hemodynamics, echocardiography, and Sonometrics crystals dimension (Sonometrics Corporation, London, Ontario, Canada) were measured at steady state and during inferior vena caval occlusion.

Results: The CardioClasp device decreased the left ventricular end-diastolic anterior-posterior dimension by $22.8 \% \pm 1.9 \%$, decreased left ventricular wall stress from $97.3 \pm 22.8$ to $67.2 \pm 7.7 \mathrm{~g} / \mathrm{cm}^{2}(P=.003)$, and increased the fractional area of contraction from $21.3 \% \pm 10.5 \%$ to $31.3 \% \pm 18.1 \%(P=.002)$. The clasp did not alter left ventricular end-diastolic pressure, left ventricular pressure, left ventricular $\mathrm{dP} / \mathrm{dt}$, or cardiac output. With the CardioClasp device, the slope of the end-systolic pressure-volume relationship was increased from $1.87 \pm 0.47$ to $3.22 \pm$ $1.55 \mathrm{~mm} \mathrm{Hg} / \mathrm{mL}(P=.02)$, the slope of preload recruitable stroke work versus end-diastolic volume was increased from $28.4 \pm 11.0$ to $44.1 \pm 23.5 \mathrm{~mm} \mathrm{Hg}(P=$ .02 ), and the slope of maximum $\mathrm{dP} / \mathrm{dt}$ versus end-diastolic volume was increased from $10.6 \pm 4.6$ to $18.6 \pm 7.4 \mathrm{~mm} \mathrm{Hg} \times \mathrm{s}^{-1} \times \mathrm{mL}^{-1}(P=.01)$. The CardioClasp device increased the slope of the end-systolic pressure-volume relationship by $68.0 \% \pm 21.7 \%$, the slope of preload recruitable stroke work versus end-diastolic volume by $50.7 \% \pm 18.1 \%$, and the slope of maximum $\mathrm{dP} / \mathrm{dt}$ versus end-diastolic volume by $85.7 \% \pm 28.9 \%$.

Conclusions: The CardioClasp device decreased left ventricular wall stress and increased the fractional area of contraction by reshaping the left ventricle. The CardioClasp device was able to maintain cardiac output and arterial pressure. The clasp increased global left ventricular contractility by increasing the slope of the end-systolic pressure-volume relationship, the slope of preload recruitable stroke work versus end-diastolic volume, and the slope of maximum $\mathrm{dP} / \mathrm{dt}$ versus enddiastolic volume. In patients with heart failure, the CardioClasp device might be effective for clinical application.

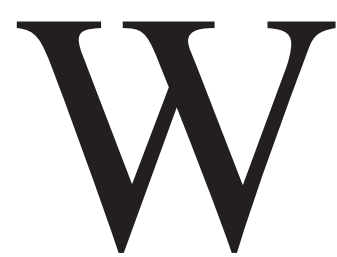

ith heart failure, left ventricular (LV) enlargement places an increased wall tension on the individual myocytes. This increased wall tension, together with cardiomyocyte dysfunction, leads to a profound depression in global LV function and contractility. If this extra geometric burden caused by ventricular dilatation can be eliminated, myocardial wall stress would decrease, leading to improved LV systolic ventricular performance. 
Melvin $^{1}$ was the first to examine the concept of passive ventricular remodeling to reduce this geometric burden. Using a computational analysis, he showed that by imposing a shape change on a dilated left ventricle, the ratio of wall tension to chamber pressure was reduced. On the basis of this concept, the CardioClasp device (CardioClasp, Inc, Cincinnati, Ohio) was developed. The CardioClasp device is a passive cardiac support device (CSD) that uses 2 indenting bars to reshape the left ventricle as 2 widely communicating lobes of reduced radius.

By passively reshaping the left ventricle and decreasing the radius of curvature, the CardioClasp device reduces LV wall stress. ${ }^{1}$ In previous studies passive reshaping has been shown to increases LV ejection fraction without altering cardiac output or systolic pressures. ${ }^{2-9}$ In the present study, using a dilated heart failure model, we tested whether the CardioClasp, by passively reshaping the left ventricle and reducing LV wall stress, can improve global LV contractility.

\section{Methods}

All study procedures were performed in compliance with the "Principles of Laboratory Animal Care" formulated by the National Society for Medical Research and the "Guide for Care and Use of Laboratory Animals" issued by the National Academy of Sciences and published by the National Institutes of Health (National Institutes of Health publication, revised 1996).

\section{Induction of Heart Failure}

After an overnight fast, 6 mongrel dogs $(22.5-26.7 \mathrm{~kg})$ were anesthetized with intravenous sodium thiopental $(15-25 \mathrm{mg} / \mathrm{kg})$ and intramuscular atropine $(0.01 \mathrm{mg} / \mathrm{kg})$, intubated, and mechanically ventilated (model R-DRAGERAV, S/N. R-2035, North American Dräger, Telford, $\mathrm{Pa}$ ). Anesthesia was maintained with $1 \%$ to $2 \%$ isoflurane (Isoflurane Vaporizer, Oharda; Isotec 3, Aushell, Ga) and oxygen. Surface electrocardiographic results (model 78346A; Hewlett Packard, Palo Alto, Calif) and oxygen saturation were monitored continuously.

As described previously, a bipolar, implantable, screw-in, transvenous myocardial pacing lead (model 4058; Medtronic, Minneapolis, Minn) was introduced through the right jugular vein and positioned at the right ventricular apex during fluoroscopy..$^{2,10}$ The pacing lead was tunneled to the back of the neck and connected to a pulse generator (THERAs model 8966i B, Medtronic) implanted subcutaneously through a 4- to 5-cm incision. The pacemaker generator was secured with the underlying muscle, and all the wounds were closed in layers.

Rapid right ventricular pacing was started on the second postoperative day. During the first week, pacing parameters were 210 ppm, 3.5-V pulse amplitude, 2.8-mV pulse sensitivity, and $1.5-\mathrm{ms}$ pulse width. The pacing rate was increased consecutively to 220 , 230, and $240 \mathrm{ppm}$ during the second, third, and fourth weeks, respectively. ${ }^{2,10}$ As in previous studies, incremental pacing reliably induced congestive heart failure manifested by clinical findings of ascites, muscle wasting, lethargy, and decreased appetite.

\section{Evaluation of the CardioClasp Device}

The heart failure animals were anesthetized with a combination of intravenous injections of diazepam $(1 \mathrm{mg} / \mathrm{kg})$, fentanyl citrate (2-8 $\mu \mathrm{g} / \mathrm{kg})$, and $2 \%$ lidocaine $(1 \mathrm{mg} / \mathrm{kg})$. The animals were ventilated through an endotracheal tube with a positive-pressure respirator (North American Drägger, model R-DRAGERAV, S/N. R-2035) and maintained with $1 \%$ to $2 \%$ isoflurane. The electrocardiogram was monitored continuously. An oxygen saturation monitor (52000 series, S/N. 9801168, WelchAllyn; Tycos Instruments Inc, Ithaca, NY) to measure the oxygen saturation was used continuously.

After administering 3000 units of heparin, a 6F micromanometer-tipped pigtail catheter (Millar Inc, Houston, Tex) was inserted and advanced to the left ventricle for measurement of the LV pressure. The side port of the catheter sheath was connected to a fluid transducer for measuring the arterial pressure. With the animal in the right lateral position, a left lateral thoracotomy was performed at the fifth intercostal space. The pericardium was opened by using a fine scissor, and the heart was suspended in a pericardial cradle. A flow probe (A-series 16-mm, S/L no.16A320; Transonic Systems Inc, Ithaca, NY) was placed around the ascending aorta and connected to a flowmeter (model T206, S/L no. 1206-S-991539, Transonic Systems Inc) to measure aortic flow.

A balloon occlusion catheter (8F, 40-mm balloon; Boston Scientific Corp, Natick, Mass) introduced through the right femoral vein was placed at the junction of the inferior vena cava (IVC) and right atrium. When required in the protocol, inflating this balloon obstructed flow in the IVC, thereby gradually decreasing cardiac output.

Sonometric crystals placement. Six small piezo electric Sonometrics crystals (Sonomicrometer Crystals; Sonometrics Inc, Ontario, Canada) were placed into the endocardial positions in the anterior, posterior, apex, base, free, and septal walls to assess LV volume (end-systolic pressure-volume relationship [ESPVR]). Crystal dimensions were measured continuously (Sonometrics Data Acquisition System, Sonometrics Inc).

Description of the CardioClasp device. The CardioClasp is an implantable medical device that consists of 3 primary components: 2 rigid bars with pads and an adjustable tether. The rigid bars have a defined curvature similar to that of the heart. Longitudinally and torsionally flexible contact pads are attached to each bar. An adjustable tether connects the 2 pad-bar assemblies on either side of the left heart.

Placement of the CardioClasp device. All pulmonary veins were mobilized from the superior to inferior aspects, and a guiding catheter was introduced underneath the pulmonary veins. The posterior bar of the CardioClasp was positioned along the long axis of the left ventricle adjacent to the posterior descending artery, and the anterior bar of the device was placed along the long axis of the left ventricle adjacent to the left anterior descending coronary artery. The anterior and posterior bars were connected with a tether to an adjustment tool. The device, once implanted, was secured to the left heart by means of mechanical fixation. Once the device was fixed, the adjustment tool pulled the tether, shortening the distance between the anterior and posterior bars and thereby decreasing the LV anterior posterior (A-P) dimension. We measured a baseline A-P end-diastolic dimension at the papillary muscle level in a short-axis echocardiographic view. After tightening the 
CardioClasp with the adjustment tool, the percentage of A-P dimension reduction was confirmed by using the short-axis echocardiographic view at the papillary muscle level.

\section{Measurements}

At baseline, the echocardiographic results, arterial and LV pressure, and aortic flow were measured, recorded, and digitized on a recorder (VIPER, Gould, Ohio). With the respirator off, these measurements were obtained under steady-state conditions and during IVC occlusions. In each experiment 3 IVC runs at baseline were recorded.

Echocardiographic measurements were obtained by using an HP Sonos 5500 machine (Hewlett Packard). All studies were performed on animals with an open chest and using an epicardial window. An s12 multifrequency transducer (Hewlett Packard) set at the midrange frequency was placed over the LV free wall by using a stand-off gel pad to obtain images. Fine epicardial sutures for repositioning marked the probe position. Two-dimensional short-axis images of the left ventricle were obtained at the level of the papillary muscle. LV internal dimensions were measured from these 2-dimensional images. End-diastolic measurements were obtained at the peak of the $\mathrm{R}$ wave on the electrocardiogram. End-systolic measurements were obtained at the end of the $\mathrm{T}$ wave.

After obtaining the baseline measurements, the CardioClasp was placed on the heart, and the LV A-P internal end-diastolic dimension was reduced by approximately $25 \%$. Echocardiograms were obtained. Hemodynamic data were acquired with the CardioClasp. The echocardiographic results, arterial and LV pressure, and aortic flow were recorded with the respirator off. These measurements were obtained under steady-state conditions and during IVC occlusions similar to the baseline measurements.

Finally, the animal was killed with $2 \mathrm{mEq} / \mathrm{kg}$ potassium chloride $(20-30 \mathrm{~mL}$ of $\mathrm{KCl})$ administered intravenously and $15 \mathrm{mg} / \mathrm{kg}$ sodium pentothal (10-15 mL) administered intravenously.

\section{Data Analysis}

Echocardiography. The echocardiographic images at end systole and end diastole were captured by the Hewlett-Packard Echocardiography machine (Sonos 5500), where the LV end-diastolic and end-systolic areas' (short-axis view) endocardial borders were traced. The dimensional and area information were used to calculate the area ejection fraction or fractional area of contraction (FAC) as follows:

$$
\mathrm{FAC}=(\text { LVEDA-LVESA }) / \text { LVEDA } \cdot 100
$$

where LVDEA is defined as LV end-diastolic area, and LVESA is defined as LV end-systolic area.

Peak LV wall stress was calculated as follows:

$$
\text { LVWS }=(0.334 \times \mathrm{LVP} \times \mathrm{LVD}) /[\mathrm{WT}(1+\mathrm{WT} / \mathrm{LVD})]
$$

where LVP is defined as LV systolic pressure, LVD is defined as $\mathrm{LV}$ anterior posterior diameter, and WT is defined as LV wall thickness. ${ }^{11,12}$

Hemodynamics. By using software developed in Visual Basic for Excel (Microsoft Excel 7.0; Microsoft Inc, Redwood, Wash), hemodynamic variables were extracted from a digitally stored data file. Ectopic and postectopic cycles were excluded from the analysis. For each cardiac cycle, the end-diastolic pressure, the peak ventricular systolic pressure, $\mathrm{LV}+\mathrm{dP} / \mathrm{dt}, \mathrm{LV}-\mathrm{dP} / \mathrm{dt}$, and peak and end-diastolic aortic pressures were determined, and stroke volume and work were calculated.

Indices of contractility. Multiple LV pressure-volume loops were obtained during transient preload reduction induced by occluding the IVC. Premature beats and subsequent beat were excluded from the analysis. LV contractility was assessed by the end-systolic pressure-volume $\left(\mathrm{P}_{\mathrm{ES}}-\mathrm{V}_{\mathrm{ES}}\right)$ relationship $\left(\mathrm{P}_{\mathrm{ES}}=\right.$ $E_{E S}\left[V_{E S}-V_{O}\right]$, where $E_{E S}$ is the slope of the ESPVR ${ }^{13}$, the preload recruitable stroke work (SW) versus end-diastolic volume $\left(\mathrm{SW}=\mathrm{M}_{\mathrm{SW}}\left[\mathrm{V}_{\mathrm{ED}}-\mathrm{V}_{\mathrm{O}, \mathrm{SW}}\right]\right)$ relationship $\left(\mathrm{M}_{\mathrm{SW}}\right),{ }^{14}$ and the maximum $\mathrm{dP} / \mathrm{dt}$ versus $\mathrm{V}_{\mathrm{ED}}\left(\mathrm{dP} / \mathrm{dt}_{\mathrm{MAX}}=\mathrm{dE} / \mathrm{dt}_{\mathrm{MAX}}\left[\mathrm{V}_{\mathrm{ED}}-\mathrm{V}_{\mathrm{O}, \mathrm{sw}}\right]\right)$ relationship. ${ }^{15}$

Data are expressed as means $\pm \mathrm{SD}$. The hemodynamic, echocardiographic, and indices data at baseline were compared with those during use of the CardioClasp by using the paired Student $t$ test.

\section{Results}

Figure 1 shows 2 representative LV short-axis echocardiographic views at the papillary muscle level at baseline and with the CardioClasp. The LV short-axis views were obtained at end diastole. With the CardioClasp, the LV A-P diameter was reduced by $25 \%$, from 4.56 to $3.43 \mathrm{~cm}$. Fractional areas of contractions were increased by $22.0 \%$. In the group data echocardiographic images revealed that the CardioClasp device reduced the end-diastolic LV A-P dimension by $22.8 \% \pm 1.9 \%$. Associated with this shape change, the CardioClasp decreased LV end-diastolic area by $20.4 \% \pm 5.0 \%$ and LV end-systolic area by $25.1 \% \pm 9.5 \%$.

Table 1 presents the absolute values for echocardiographic parameters at baseline and with the CardioClasp. With the CardioClasp, end-systolic and end-diastolic LV A-P and septal free wall thickness were increased slightly. End-diastolic septal free wall dimension were unchanged, whereas end-systolic septal free wall dimensions were increased $(P=.022)$.

With the CardioClasp (Figure 2), myocardial LV wall stress decreased significantly from baseline values $(97.3 \pm$ 22.8 to $\left.67.2 \pm 7.7 \mathrm{~g} / \mathrm{cm}^{2}, P=.003\right)$. The CardioClasp decreased myocardial LV wall stress by $28.9 \% \pm 4.4 \%$ compared with baseline values.

With the CardioClasp (Figure 3), the FAC increased significantly from baseline $(21.3 \pm 10.5$ to $31.3 \pm 18.1$, $P=.002$ ). The CardioClasp increased FAC significantly by $50.9 \% \pm 18.1 \%$ compared with baseline values.

From one experiment, Figure 4 shows different indices of LV contractility. In Figure 4, A, LV pressure-volume loops are plotted at baseline and with the CardioClasp together with the ESPVRs. In this example the CardioClasp increased the slope of the ESPVR. Figure 4, A, shows one example of ESPVR loops generated during the baseline period and with the CardioClasp at $25 \%$. The data show a 


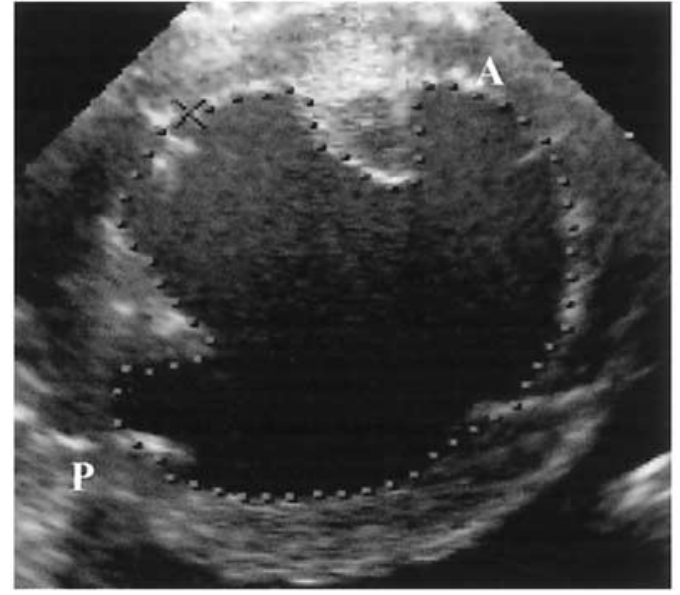

Baseline at end-diastole

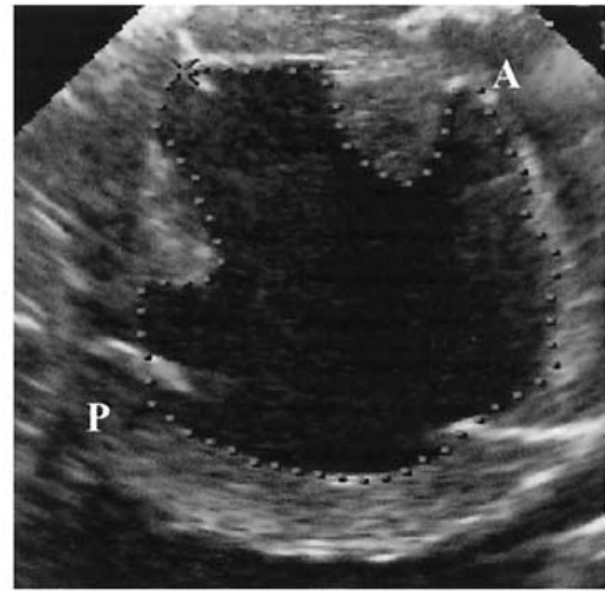

Clasp at end-diastole

Figure 1. Two representative LV end-diastolic short-axis echocardiographic views at papillary muscle level at baseline heart failure and with the CardioClasp. The CardioClasp significantly reduced LV A-P diameter by $25 \%$. The figure shows how the A-P dimension was measured connecting the line immediately below to the anterior and posterior papillary muscles of the left ventricle $(A$, corner point of the anterior papillary muscle; $P$, corner point of the posterior papillary muscle). In this image the anterior bar of the CardioClasp device was in front of the anterior papillary muscle and not visible. The posterior bar of the Cardioclasp device was at the back of the posterior papillary muscle and poorly visible (slightly echogenic behind the papillary muscle).

TABLE 1. Effects of the CardioClasp on echocardiographic parameters

\begin{tabular}{|c|c|c|c|c|}
\hline & LV echocardiographic parameters & Baseline & CardioClasp & $P$ value \\
\hline \multirow[t]{5}{*}{ Diastole } & A-P dimension $(\mathrm{cm})$ & $4.31 \pm 0.34$ & $3.39 \pm 0.34^{*}$ & .0001 \\
\hline & S-FW dimension (cm) & $4.04 \pm 0.34$ & $4.04 \pm 0.42$ & .488 \\
\hline & A-P wall thickness (mm) & $9.6 \pm 2.1$ & $9.7 \pm 1.3$ & .431 \\
\hline & S-FW wall thickness (mm) & $9.4 \pm 1.7$ & $9.5 \pm 1.1$ & .485 \\
\hline & End-diastolic area $\left(\mathrm{cm}^{2}\right)$ & $12.2 \pm 2.1$ & $9.7 \pm 2.3^{*}$ & .007 \\
\hline \multirow[t]{5}{*}{ Systole } & A-P diameter $(\mathrm{cm})$ & $3.73 \pm 0.36$ & $3.10 \pm 0.44^{*}$ & .0001 \\
\hline & S-FW dimension (cm) & $3.70 \pm 0.55$ & $3.92 \pm 0.54^{*}$ & .022 \\
\hline & A-P wall thickness (mm) & $10.0 \pm 1.7$ & $11.1 \pm 2.2^{*}$ & .040 \\
\hline & S-FW wall thickness (mm) & $10.1 \pm 1.6$ & $11.3 \pm 2.2$ & .052 \\
\hline & End-systolic area $\left(\mathrm{cm}^{2}\right)$ & $10.8 \pm 2.6$ & $8.0 \pm 3.1^{*}$ & .034 \\
\hline
\end{tabular}

$S-F W$, Septal free wall.

${ }^{*} P<.05$.

steepening of the LV ESPVR slope (increased $\mathrm{E}_{\max }$ ) at $25 \%$.

Figure $4, B$, shows the effects of the CardioClasp on preload recruitable stroke work. The rounded bullets and solid line represent the SW-EDV relationship with the CardioClasp and the dotted line with solid bullets represents the SW-EDV relationship at baseline. Clearly, the CardioClasp increased the SW-EDV relationship compared with baseline values.

Figure 4, $C$, shows the effects of the CardioClasp on $\mathrm{dE} / \mathrm{dt}$, the slope of the maximum $\mathrm{dP} / \mathrm{dt}-\mathrm{V}_{\mathrm{ED}}(\mathrm{dP} /$ $\mathrm{dt}_{\mathrm{MAX}}-\mathrm{V}_{\mathrm{ED}}$ ) relationship. The rounded bullets and solid line represent $\mathrm{dE} / \mathrm{dt}$ with the CardioClasp, and the dotted line with solid bullets represents $\mathrm{dE} / \mathrm{dt}$ at baseline. The CardioClasp increased $\mathrm{dE} / \mathrm{dt}$ compared with baseline values.

Table 2 presents the absolute hemodynamic parameters at baseline and with the CardioClasp. With the CardioClasp, cardiac output was unaltered $(P=.40)$. Likewise, peak LV systolic $(P=.26)$ and $\mathrm{LV}$ end-diastolic $(P=.43)$ pressures, peak positive $(P=.12)$ and negative $(P=.23)$ first derivatives of $\mathrm{LV}$ pressures $(\mathrm{dP} / \mathrm{dts})$, and aortic systolic $(P=.38)$ and diastolic $(P=.12)$ pressures were unaltered.

$\mathrm{LV}$ contractility, assessed by means of $\mathrm{E}_{\mathrm{ES}}, \mathrm{M}_{\mathrm{SW}}$, and $\mathrm{dE} / \mathrm{dt}_{\mathrm{MAX}}$, was significantly greater in the CardioClasptreated group than in the baseline heart failure group. Table 


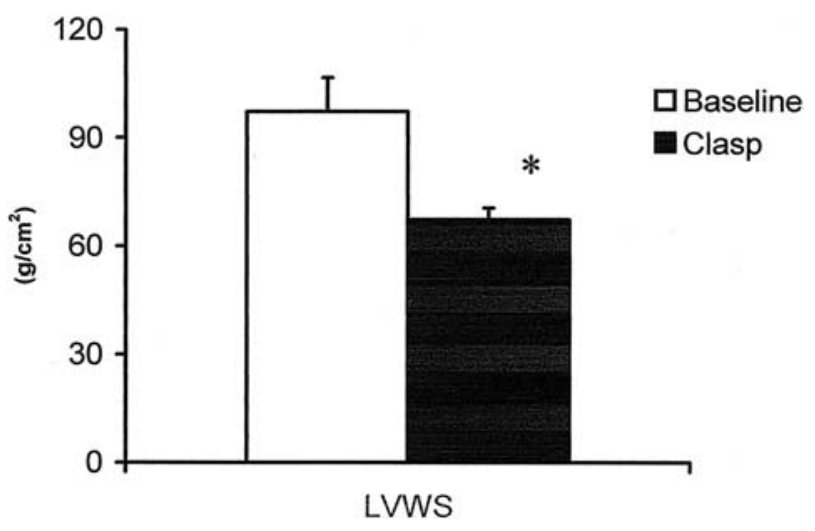

Figure 2. Significantly decreased LV wall stress (LVWS) with the CardioClasp by $28.9 \% \pm 4.4 \%$. ${ }^{*} P<.05$.

3 shows the systolic indices of the pressure-volume relationship at baseline and with the CardioClasp. With the CardioClasp, $\mathrm{E}_{\max }$ increased by $68.0 \% \pm 21.7 \%(P=.02)$, preload recruitable stroke work increased by $50.7 \% \pm$ $18.1 \%(P=.02)$, and $\mathrm{dE} / \mathrm{dt}$ increased by $85.7 \% \pm 28.9 \%$ $(P=.01)$.

\section{Discussion}

The concept and understanding of passive LV geometric reshaping is evolving. Melvin ${ }^{1}$ was the first to examine the concept of passive geometric ventricular remodeling by using a computational analysis. The model showed that by imposing a shape change on a dilated left ventricle, the ratio of wall tension to chamber pressure was reduced. If the reduction in wall tension occurred as a result of ventricular resection, then a substantial degree of contractile shortening improvement would be needed just to maintain baseline stroke volume.

Shimizu and colleagues ${ }^{3}$ examined the effects of passive LV reshaping in a canine model of dilated heart failure. After rapid ventricular pacing induced heart failure, the heart was attached to an isolated heart preparation. The CardioClasp was positioned on the heart and adjusted to reduce A-P end-diastolic dimension by $20 \%$ to $30 \%$. With the CardioClasp, the ESPVR was significantly increased from $1.4 \mathrm{~mm} \mathrm{Hg} / \mathrm{mL}$ at baseline to $2.4 \mathrm{~mm} \mathrm{Hg} / \mathrm{mL}$. After taking off the device, ESPVR returned to the baseline level $(1.5 \mathrm{~mm} \mathrm{Hg} / \mathrm{mL})$. Shimizu and colleagues also showed that both the systolic and diastolic pressures and the developed pressure decreased with rapid removal of the CardioClasp.

Earlier, our group reported acute hemodynamic improvements with the CardioClasp at approximately $30 \%$ and $15 \%$ $\mathrm{LV}$ end-diastolic dimension reduction. ${ }^{2}$ We showed LV dimension reduction at $31.3 \%$, and the FAC was increased by $27 \%$.

Santamore and coworkers ${ }^{4}$ reported that at normal afterload, the CardioClasp maintained the stroke volume versus

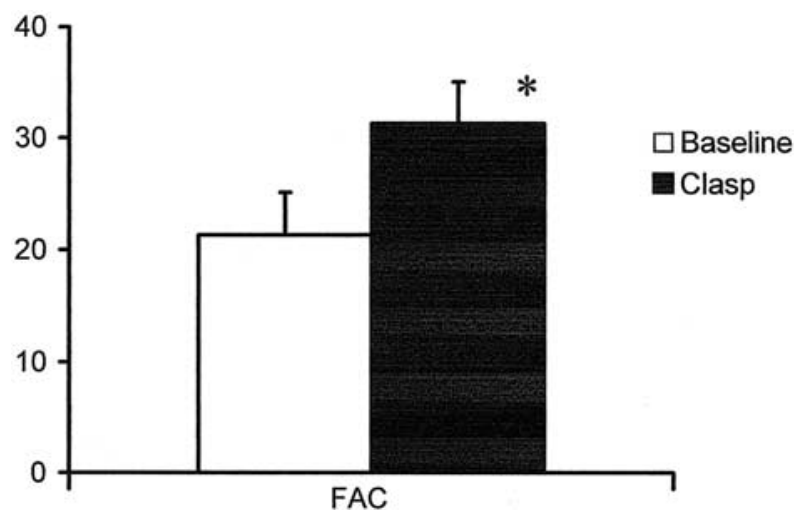

Figure 3. Significantly increased LV FAC by $50.9 \% \pm 18.1 \%$ with the CardioClasp. ${ }^{*} \boldsymbol{P}<.05$.

end-diastolic pressure relationship. At increased afterload, the decrease in the stroke volume versus end-diastolic pressure relationship was less with the CardioClasp. Santamore and coworkers also reported that LV reshaping with the CardioClasp attenuated the cardiac output decrease in response to increased afterload. With increased afterload, the stroke volume decreased significantly less with the CardioClasp $(-20.4 \% \pm 0.4 \%)$ compared with control values $(-27.7 \% \pm 0.6 \%, P<.05)$.

In our experiments the CardioClasp acutely reduced the LV A-P diameter by $22.8 \%$, as demonstrated by means of direct epicardial transthoracic echocardiography. The CardioClasp device also significantly reduced the LV systolic wall stress by $29 \%$. Our measurement of FAC clearly showed the effective results with the passive CardioClasp device; the FAC was increased by $51 \%$ with the CardioClasp at implantation. In these experiments the CardioClasp increased $\mathrm{E}_{\max }$ by $68.0 \%, \mathrm{M}_{\mathrm{sw}}$ by $50.7 \%$, and $\mathrm{dE} / \mathrm{dt}_{\mathrm{MAX}}$ by $85.7 \%$, indicating improved LV myocardial contractility.

Baseline LV systolic and diastolic pressures were not significantly altered compared with pressures with use of the CardioClasp. Likewise, cardiac output was unaltered with use of the CardioClasp at implantation $(1.9 \mathrm{~L} / \mathrm{min})$ compared with baseline heart failure. The reason for increased ejection fraction and contractility with unaltered cardiac output $(1.9 \mathrm{~L} / \mathrm{min})$ probably lies in the decreased LV end-diastolic volume, as evidenced by the decreased enddiastolic areas after CardioClasp placement.

The key findings of the present study are the following:

1. The CardioClasp reshaped the left ventricle, thereby reducing the LV systolic wall stress.

2. The FAC increased, whereas cardiac output and LV pressure were unaltered.

3. The CardioClasp significantly increased global LV contractility, as indicated by the increases in $\mathrm{E}_{\max }$, $\mathrm{M}_{\mathrm{SW}}$, and the slope of the maximum $\mathrm{dP} / \mathrm{dt}-\mathrm{V}_{\mathrm{ED}}(\mathrm{dE} /$ $\mathrm{dt}_{\mathrm{MAX}}$ ) relationship. 



Figure 4. A, Representative recordings showing LV pressure-volume loops at baseline heart failure and during CardioClasp placement at $25 \%$ LV dimension reduction. LV contractility assessed by $E_{\max }$ was significantly higher by $68.0 \% \pm 21.7 \%$ with the CardioClasp ( $P=.01$ compared with baseline heart failure). $L V V$, LV volume; LVP, LV pressure. B, Preload recruitable stroke work $(S W)$ versus end-diastolic volume (EDV) relationship at baseline heart failure (solid bullets and dotted line) and with the CardioClasp (rounded bullets and solid line). The CardioClasp significantly increased the preload recruitable stroke work-end-diastolic volume relationship $(P=.02)$. $M_{s w}$ Slope of the LV preload recruitable stroke work (in millimeters of mercury); EDV, LV end-diastolic volume (in millimeters). C, Slope of the maximum $\mathrm{dP} / \mathrm{dt}-\mathrm{V}_{\mathrm{ED}}\left(\mathrm{dP}_{\mathrm{d}} \mathrm{dt}_{\mathrm{MAX}}-\mathrm{V}_{\mathrm{ED}}\right)$ relationship (dE/dt) at baseline heart failure (solid bullets and dotted line) and with the CardioClasp (rounded bullets and solid line). The CardioClasp significantly increased $\mathrm{dE} / \mathrm{dt}$ (in millimeters of mercury per second per milliliter), $(P=.02) . L V d P / d t$, First derivative of the LV pressure (in millimeters or mercury per second); EDV, LV end-diastolic volume (in milliliters).

Comparison with the Literature

The Myosplint device pulls the opposing walls of the heart together, thereby decreasing the chamber radius. Each Myosplint implant consists of 2 epicardial buttons connected by a tension member that bisects the left ventricle. The Myosplint buttons (generally 3 sets) are placed down the long LV axis to create a bilobular shape..$^{5-7}$ In initial experimental studies Takagaki and associates ${ }^{6}$ confirmed acute LV shape changes induced by the Myosplint and showed that this passive device was associated with a $20 \%$ decrease in wall stress and an increase in LV ejection fraction. Cardiac output and ventricular pressures were unaltered. These benefits were maintained over 30 days. ${ }^{5}$ Peak LV elastance and preload recruitable stroke work increased from baseline to 4 weeks after placing the Myosplint. ${ }^{7}$

In human subjects the Myosplint was initially tested in patients awaiting heart transplantation. Placed just before removing the heart, this passive device decreased peak LV systolic wall stress. ${ }^{8}$ In an initial clinical series the device was implanted in 5 patients in New York Heart Association class III with dilated cardiomyopathy. ${ }^{8}, 9$ Placement of the Myosplints was safely performed without early significant 
TABLE 2. Effects of the CardioClasp on hemodynamic parameters

\begin{tabular}{lcc}
\hline Hemodynamic parameters & Baseline heart failure & CardioClasp \\
\hline LVEDP $(\mathrm{mm} \mathrm{Hg})$ & $17.5 \pm 3.1$ & $17.9 \pm 4.3$ \\
LVSP $(\mathrm{mm} \mathrm{Hg})$ & $80.3 \pm 12.4$ & $82.4 \pm 16.9$ \\
Max dP/dt $(\mathrm{mm} \mathrm{Hg} / \mathrm{s})$ & $782 \pm 223$ & $956 \pm 519$ \\
Min dP/dt $(\mathrm{mm} \mathrm{Hg} / \mathrm{s})$ & $-700 \pm 151$ & $-786 \pm 291$ \\
AoPsys $(\mathrm{mm} \mathrm{Hg})$ & $78.4 \pm 12.0$ & $79.5 \pm 16.6$ \\
AoPdias $(\mathrm{mm} \mathrm{Hg})$ & $52.0 \pm 4.6$ & $58.4 \pm 13.4$ \\
CO (L/min) & $1.9 \pm 0.4$ & $1.9 \pm 0.5$
\end{tabular}

$\angle V E D P$, Left ventricular end-diastolic pressure; $L V S P$, left ventricular peak systolic pressure; Max $d P / d t$, peak first positive derivative of the left ventricular pressure; $M i n d P / d t$, peak first negative derivative of the left ventricular pressure; AoPsys, peak systolic aortic pressure; AoPdias, peak diastolic aortic pressure; $\mathrm{CO}$, cardiac output.

adverse events. However, reconfiguration of the left ventricle by the Myosplints might necessitate simultaneous mitral valve repair in patients with clinically significant incompetence. ${ }^{16}$

\section{Passive Cardiac Restraint}

The results of cardiomyoplasty have led to the development of passive devices to constrain the heart and prevent further ventricular dilatation. ${ }^{17,18}$ One such device is the Acorn CorCap CSD. The Acorn CorCap CSD is a mesh-like jacket that is slipped around the heart and adjusted to prevent the heart from further dilatation.

Most experimental studies with the Acorn device have involved induction of heart failure, placement of the device, and then, weeks to months later, examination of ventricular function. In an experimental study Chaudhry and coworkers ${ }^{19}$ produced heart failure by means of intracoronary microembolization. In the control animals LV size continued to increase, and ejection fraction decreased. In the Acornsupported animals, LV size decreased slightly, and ejection fraction increased. Sabbah and associates ${ }^{20}$ showed that the percentage of myocyte shortening and the peak velocity of shortening $(\mathrm{dS} / \mathrm{dt})$ were significantly increased in CSDtreated dogs undergoing heart failure. In a rapid ventricular pacing heart failure model, Power and colleagues ${ }^{21}$ reported higher LV fractional shortening and less mitral regurgitation in Acorn-support animals. In sheep 1 week after coronary artery ligation, Pilla and associates ${ }^{22,23}$ placed the Acorn CSD around the heart. At terminal study, infarct size was significantly smaller, and ventricular function was significantly better in the Acorn-supported animals.

In patients with idiopathic cardiomyopathy, Konertz and coworkers ${ }^{24}$ reported a decrease in LV end-diastolic and LV end-systolic diameters and an increase in LV ejection fraction by using the Acorn device in patients. These benefits were maintained at 1 year. ${ }^{24,25}$ Six patients with symptomatic heart failure caused by ischemic cardiomyopathy were
TABLE 3. Effects of the CardioClasp on LV contractility

\begin{tabular}{llll}
\hline & \multicolumn{1}{c}{$\mathbf{E}_{\max }$} & \multicolumn{1}{c}{$\mathbf{M}_{\mathbf{s w}}$} & \multicolumn{1}{c}{$\mathbf{d E} / \mathbf{d t}_{\text {max }}$} \\
$(\mathbf{m m ~ H g} / \mathbf{m L})$ & \multicolumn{1}{c}{$\mathbf{m m ~ H g}$} & $\left(\mathbf{m m ~ H g} \cdot \mathbf{s}^{-\mathbf{1}} \cdot \mathbf{m L}\right)$ \\
\hline Baseline & $1.87 \pm 0.47$ & $28.4 \pm 11.0$ & $10.60 \pm 4.58$ \\
Clasp & $3.22 \pm 1.55^{*}$ & $44.1 \pm 23.5^{*}$ & $18.58 \pm 7.42 \dagger$ \\
\hline
\end{tabular}

$E_{\text {max }}$ Slope of LV ESPVR; $M_{S W}$, slope of preload recruitable stroke work versus end-diastolic volume; $d E / d t_{\text {max }}$ slope of the maximum $d P / d t-V_{E D}$ $\left(\mathrm{dP} / \mathrm{dt}_{\max }-\mathrm{V}_{\mathrm{ED}}\right)$ relationship.

${ }^{*} P=0.02$ and $\dagger P=0.01$ when compared with baseline values.

treated surgically with the Acorn CSD and underwent coronary artery bypass grafting. ${ }^{24-26}$ Ventricular reconstruction was also performed in 5 of the 6 patients. Both the LV end-diastolic and LV end-systolic diameters were significantly decreased 1 month postoperatively and did not change significantly over the next 11 months. Mitral regurgitation improved from a mean of 2.7 preoperatively to 1.4 at 12 months, and the average LV ejection fraction also increased from $27 \%$ preoperatively to $35.9 \%$ at 12 months after surgical intervention. ${ }^{25}$

\section{Limitations of the Study}

Despite the fact that we used a dilated cardiomyopathy animal model, the mechanism and dilatation in pacinginduced cardiomyopathy is different than that in the human idiopathic cardiomyopathy and poorly understood. Further chronic studies are to be carried out to understand the chronic changes and effects of CardioClasp device placement on dilated cardiomyopathy. Currently, at Temple University, we have started chronic studies with a pacing model, in which the CardioClasp is implanted for 30 days. After device implantation, the pacing parameters are continued to maintain the heart failure model. We reported a preliminary report ${ }^{27}$ of chronic data as an abstract $(\mathrm{n}=4)$ at the 2002 American Society for Artificial Internal Organs meeting. After 30 days of chronic implantation, the epicardial area underneath the anterior and posterior bars showed fibrous tissue formation underneath the pads. However, histologic epicardial and myocardial samples did not show any ischemic changes related to the pads. No necrosis or myocardial injury was present at the compression sites by the bars of the CardioClasp device. As the numbers of animals were few, further randomized chronic studies with sham operations are to be carried out for statistical differences. These results can be used to focus on the effects of the CardioClasp in dilated cardiomyopathy and might be closer to those of surgical intervention for clinical dilated cardiomyopathy.

\section{Summary and Conclusions}

The goal of medical management of heart failure is to reduce afterload. This is usually achieved by lowering vascular resistance. The resulting lower arterial blood pressure 
can compromise blood flow to other vital organs, such as the brain and kidney. The CardioClasp device can reduce the tension on the myocardial cells without decreasing arterial blood pressure. This leads to improved LV systolic performance. The CardioClasp significantly increased the stroke work versus end-diastolic volume relationship and the slope of the maximum $\mathrm{dP} / \mathrm{dt}-\mathrm{V}_{\mathrm{ED}}\left(\mathrm{dE} / \mathrm{dt}_{\mathrm{MAX}}\right)$ relationship, and $\mathrm{E}_{\max }$ was also significantly increased by the CardioClasp. With overall increased LV systolic indices and contractility, this passive geometric ventricular reshaping has the added potential for preserving the contractile mass and circumferential length.

We thank Dr Milton April and Lewis Bright for their dedicated support and animal care for this experiment.

\section{References}

1. Melvin DB. Ventricular radius reduction without resection: a computational analysis. ASAIO J. 1999;45:160-5.

2. Kashem MA, Santamore WP, Hassan S, Crabbe DL, Margulies KB, Melvin DB. CardioClasp ${ }^{\mathrm{TM}}$ : a new passive device to re-shape cardiac enlargement. ASAIO J. 2002;48:253-9.

3. Shimizu J, Wang J, Yi GH, He K, Kashem MA, Crabbe DL, et al. Improved systolic performance by passive remodeling in experimental heart failure. Circulation. 2000;102(suppl 18):683-3304.

4. Santamore WP, Kashem A, Hassan S, Crabbe DL, Margulies KB, Melvin DB. Left ventricular reshaping enhances contractility and response to increased afterload. Presented at the 8th Annual Meeting of Cardiac Technology and Transfer with STS meeting; 2002 Feb; Fort Lauderdale, Fla. Promedica International, a California Corporation.

5. McCarthy P, Takagaki M, Ochiai Y, Young JB, Tabata T, Shiota T, et al. Device-based change in left ventricular shape: a new concept for the treatment of dilated cardiomyopathy. J Thorac Cardiovasc Surg. 2001;122:482-90.

6. Takagaki M, Fukamachi K, McCarthy P, Ochiai Y, Dessoffy R, Vidlund R, et al. Novel device to change left ventricular shape for heart failure treatment: device design and implantation procedure. ASAIO J. 2001;47:244-8.

7. McCarthy P, Fukamachi K, Takagaki M, Armstrong G, Young J, Schweich C, et al. Device based left ventricular shape change immediately reduces left ventricular volume and increases ejection fraction in a pacing induced cardiomyopathy model in dogs: a pilot study [abstract]. J Am Coll Cardiol. 2000;35:183A.

8. Fukamachi K. Myosplint: a new device to treat heart failure by changing left ventricular shape. Proceedings of the 4th International Symposium on Volume Reduction Surgery. J Card Surg. 2002:16.

9. Fukamachi K, McCarthy P, Takagaki M, Ochiai Y, Doi K, Dessoffy $\mathrm{R}$, et al. Myosplint improved left ventricular function by improving systolic elastance and maintaining diastolic compliance in a canine cardiomyopathy model. Circulation. 2001;104:II-440.

10. Shannon RP, Komamura K, Stamber BS, Manders WT, Vatner SF. Alterations in left ventricular geometry and myocardial contractility in conscious dogs with pacing induced dilated cardiomyopathy. Am J Physiol. 1991;260:H1903-11.

11. Mirsky I, Pasipoularides A. Clinical assessment of diastolic function. Prog Cardiovasc Dis. 1990;32:291-318.

12. Spinale FG, Coker ML, Krombach SR, Mukherjee R, Hallak H, Houck WV, et al. Matrix metalloproteinase inhibition during the development of congestive heart failure: effects on left ventricular dimensions and function. Circ Res. 1999;85:364-76.

13. Sagawa K. The end-systolic pressure-volume relation of the ventricle: definition, modifications, and clinical use. Circulation. 1981;63:1223-7.

14. Glower DD, Spratt JA, Snow ND, Kabas JS, Davis JW, Olsen CO, et al. Linearity of the Frank-Starling relationship in the intact heart: the concept of preload recruitable stroke work. Circulation. 1985;71:9941009.

15. Little WC. The left ventricular $\mathrm{dP} / \mathrm{dt}$ max end-diastolic volume relation in closed-chest dogs. Circ Res. 1985;56:808-15.

16. Schenk S, Reichenspurner H, Groezner JG, Boehm DH, Schirmer J, Scheidt WV, et al. Myosplint implantation and ventricular shape changes in patients with dilative cardiomyopathy-first clinical experience. J Heart Lung Transplant. 2001;20:217.

17. Patel HJ, Polidori DJ, Pilla JJ. Stabilization of chronic remodeling by asynchronous cardiomyoplasty in dilated cardiomyopathy: effects of a conditioned muscle wrap. Circulation. 1997;96:3665-71.

18. Kass DA, Baughman KL, Pak PH, Cho PW, Levine HR, Gardner TJ, et al. Reverse remodeling from cardiomyoplasty in human heart failure. External constraint versus active assist. Circulation. 1995;91: 2314-8.

19. Chaudhry PA, Mishima T, Sharov VG, Hawkins J, Alferness CA, Paone G, et al. Passive epicardial containment prevents ventricular remodeling in heart failure. Ann Thorac Surg. 2000;70:1275-80.

20. Sabbah HN, Chaudhry PA, Paone G, Mishima T, Alferness C. Passive ventricular constraint with the Acorn prosthetic jacket prevents progressive left ventricular dilation and improves ejection fraction in dogs with moderate heart failure [abstract]. J Am Coll Cardiol. 1999;33: 207A.

21. Power JM, Raman J, Dornom A, Farish SJ, Burrell LM, Tonkin AM, et al. Passive ventricular constraint amends the course of heart failure. Cardiovasc Res. 1999;44:549-55.

22. Pilla JJ, Blom AS, Brockman DJ, Bowen F, Yuan Q, Giammarco J, et al. Ventricular constraint using the Acorn cardiac support device (CSD) limits infarct expansion. Circulation. 2001;104:II-480.

23. Pilla JJ, Brockman DJ, Blom AS, Bowen F, Yuan Q, Gorman JH, et al. Prevention of dilation using the Acorn cardiac support device (CSD) results in reverse remodeling and improvement of function. Circulation. 2001;104:II-440.

24. Konertz WF, Shapland JE, Hotz H, Dushe S, Braun JP, Stantke K, et al. Passive containment and reverse remodeling by a novel textile cardiac support device. Circulation. 2001;104(suppl I):I-270-5.

25. Konertz WF, Kleber FX, Dushe S, Hotz H, Stantke K. Efficacy trends with the Acorn Cardiac Support Device: One year follow-up. Circulation. 2001;104:II-357.

26. Raman J, Power JM, Buxton BF, ALferness CA, Hare D. Ventricular containment as an adjunctive procedure in ischemic cardiomyopathy: early results. Ann Thorac Surg. 2000;70:1124-6.

27. Kashem A, Hassan S, Santamore WP, Crabbe DL, Margulies KB, Goldman BI, et al. Chronic experimental heart failure: CardioClasp ${ }^{\mathrm{TM}}$ can maintain improved myocardial function [abstract]. J ASAIO. 2002; $48: 161$.

\section{Discussion}

Dr W. Randolph Chitwood (Greenville, NC). Dr Kashem, I think you have done an eloquent job in studying physiologically the changes associated with these new devices, which we hope will provide great improvement in hemodynamics for patients with these terribly dilated ventricles. They really had no hope in the past. We congratulate you on your work. I have several questions.The first question is, how is this device incorporated into heart tissue? Did you do any studies looking at the incorporation of the device into the myocardium?

Dr Kashem. We checked with the histology from chronic and acute studies, and we did not find any problem with the histology, and there was no significant abrasion incorporating the device over to the myocardium. Histologically, it did not show any gross abnormalities of the myocardium.

Dr Chitwood. After this device is working and active, does it change papillary muscle function, tip distance, or angulation with the leaflets through the chords? Was there any mitral insufficiency seen in these patients? 
Dr Kashem. No, we did not present those date here, but at the International Society for Heart and Lung Transplantation meeting, I presented the mitral regurgitation. We performed transesophageal echocardiography in all the animal studies, which we did not show here, and I found there are no changes in mitral regurgitation. Mostly in heart failure we found that mitral regurgitation was grade 1 to 2 in most cases, and we found there was no significant alteration of the mitral regurgitation in those cases.Regarding papillary muscle, I did not see any implications with the CardioClasp device.

Dr Chitwood. Do you think this device could actually be used in a different way to reorient papillary muscles in patients who have ischemic mitral regurgitation or cardiomyopathic mitral regurgitation? Can you reorient the chordae? Could this device decrease mitral insufficiency?

Dr Kashem. We did not investigate the means of the placement. We did investigate several ways to place the device, and we did not find any significant changes. But maybe in the future we can see whether there are any significant effects on the papillary muscle or mitral regurgitation by placing the device.

Dr Chitwood. How does this device differ from the Myocor and Acorn devices in its efficacy?
Dr Kashem. The basic theory for the Myocor and CardioClasp is the same-to reduce wall stress-but the important thing here is that it is a non-blood contact device for CardioClasp. It does not penetrate the left ventricle. The other one has a bilobular shape, and the CardioClasp does the same thing. Compared with the Acorn device, the CardioClasp device has an immediate effect on contractility and an immediate effect on reduction of the wall stress and tension on the myocardial cells. It should have a reverse remodeling in long-term cases; we did not study those cases. I guess a 3-month or 6-month study could answer the question of whether there is a reverse remodeling at the myocyte level.

Dr Chitwood. It would be interesting to compare these devices as to their efficacy and efficiency using your hemodynamic models.

Dr Kashem. Yes.

Dr Chitwood. What is the ideal target patient population for these devices?

Dr Kashem. Mostly patients undergoing dilated cardiomyopathy of ischemic or nonischemic origin. The CardioClasp device could be placed in New York Heart Association class III or IV patients. But we still have to go further on the inclusion-exclusion criteria. We did not decide that.

\section{Authoritative}

The Journal of Thoracic and Cardiovascular Surgery is the most frequently cited thoracic/cardiovascular surgery journal in the Science Citation Index. An article in JTCVS is cited on average almost twice as often as those in the closest cardiothoracic journal. 